\title{
Une gamma-caméra miniaturisée pour la cancérologie
}

Stéphanie Pitre-Champagnat (stephanie.pitre@u-psud.fr)

Laboratoire IR4M, UMR 8081, Institut Gustave Roussy, 114 rue Édouard Vaillant, 94805 Villejuif Cedex

En cancérologie, de nouveaux systèmes d'imagerie médicale conçus pour améliorer

le diagnostic et guider

\section{la thérapie ont bénéficié}

des avancées technologiques

en instrumentation nucléaire.

C'est le cas pour

le développement des

détecteurs de radioactivité

compacts utilisés en bloc

opératoire pour guider

le chirurgien en temps réel

vers les tumeurs préalablement

radiomarquées, diminuant

ainsi le risque d'une ablation

incomplète.

La gamma-caméra appelée

POCI a été I'un des premiers

dispositifs d'imagerie développé

pour l'assistance au traitement

chirurgical du cancer, et a été

évaluée récemment sur

une cohorte de 162 patientes

présentant un cancer du sein.

Les termes marqués d'un astérisque sont définis dans le glossaire, p. 45 .
Le succès du traitement chirurgical du cancer, qui est actuellement le protocole thérapeutique le plus largement utilisé, repose sur l'identification précise et la résection complète des tissus pathologiques. Cette intervention peut rapidement devenir délicate lorsque la tumeur est mal délimitée, peu accessible ou bien encore de petite taille.

Plusieurs stratégies sont donc employées aujourd'hui pour faciliter l'ablation tumorale comme, par exemple, la localisation de certaines structures à l'aide d'un colorant ou d'un radio-isotope associé à une molécule sensible à la physiologie ou au métabolisme de la tumeur. Dans ce dernier cas, on parle de traitement chirurgical radioguidé où le chirurgien, muni d'une sonde de comptage de la radioactivité, est guidé vers les lésions préalablement radio-marquées. Cette méthode de détection per-opératoire ${ }^{\star}$ permet à la fois de simplifier l'accès à un foyer tumoral, de fournir un facteur discriminant supplémentaire entre tissus sains et tissus tumoraux, et de découvrir parfois des sites pathologiques passés inaperçus lors $\mathrm{du}$ bilan diagnostique. Cette technique d'assistance chirurgicale par radioguidage est aujourd'hui une pratique standard en cancérologie, où la résection complète des tissus pathologiques est indispensable pour envisager la rémission du patient.

Cependant, l'expérience clinique a mis en évidence des échecs de détection répétés avec les sondes de comptage, correspondant à des situations anatomiques et physiologiques particulières, comme celles rencontrées lorsque la spécificité du traceur radioactif est faible ou encore lorsque plusieurs foyers de fixation figurent dans le champ de détection du compteur de radioactivité. Ces limitations, inhérentes à la technique de comptage, peuvent être comblées dans la plupart des cas en ayant recours à un système de détection réalisant l'image radioactive de la plaie opératoire. C'est dans ce contexte que les gamma-caméras miniaturisées ont été développées.

\section{L'imagerie gamma per-opératoire pour le traitement chirurgical du cancer}

Les dispositifs d'imagerie gamma miniaturisés sont conçus sur le même principe que les gamma-caméras standards, mais leur développement a longtemps été freiné par les dimensions encombrantes des photodétecteurs disponibles. En plus de leur compacité et d'un poids réduit assurant la bonne maniabilité de l'appareil, ces systèmes de radio-imagerie doivent répondre à plusieurs critères de détection imposés par les conditions du radioguidage per-opératoire. D'une part, l'efficacité de détection doit être optimisée en vue d'obtenir des temps de prise d'image aussi brefs que possible pour ne pas retarder l'intervention chirurgicale. D'autre part, la résolution énergétique doit être la meilleure possible pour minimiser les effets de la diffusion des photons gamma dans les tissus environnants, et ainsi ne pas les visualiser sur les images des lésions tumorales radiomarquées. Enfin, une résolution spatiale millimétrique associée à une faible distorsion de l'image sont nécessaires pour permettre la distinction de plusieurs foyers radioactifs dans la plaie opératoire.

En tenant compte des conditions d'utilisation et des performances de détection recherchées, plusieurs groupes de recherche académique en Amérique du Nord, Japon, Italie, Espagne et France, ainsi que quelques 
La sonde POCl (Per-Operative Compact Imager), caméra miniature haute résolution comportant un dispositif détectant les rayons gamma et utilisée en bloc opératoire pour cibler des lésions tumorales préalablement marquées de manière radioactive.

industriels, ont développé des gammacaméras miniaturisées, spécifiquement dédiées à la détection per-opératoire. On dénombre à ce jour dix dispositifs commercialisés et neuf autres développés au sein des laboratoires de recherche. Tous sont composés à l'image d'une gamma-caméra standard, c'est-à-dire d'un collimateur couplé à un système à localisation gamma.

Un collimateur multicanaux, généralement en plomb, permet de sélectionner la direction des rayonnements gamma incidents. Il conditionne ainsi la sensibilité du dispositif qui est comprise entre 0,005\% (i.e., 5 photons détectés pour 100000 émis) et $0,2 \%$ à une énergie gamma de $140 \mathrm{keV}$, ce qui permet des acquisitions d'image en quelques secondes. Pour augmenter cette efficacité de détection, certaines caméras sont pourvues de collimateurs sténopés ${ }^{\star}$, dits "pinholes", qui conduisent à d'excellentes performances de détection lorsque la caméra est située à moins de $3 \mathrm{~cm}$ de la lésion radiomarquée. En contrepartie, ces collimateurs pinholes entraînent des effets de grandissement selon la profondeur de l'objet d'intérêt par rapport à la surface du collimateur, et compliquent ainsi l'interprétation des images.

Le système de localisation repose soit sur la mise en ouvre d'un détecteur à semiconducteur, soit sur un scintillateur couplé à un photodétecteur à faible encombrement. Les imageurs basés sur les semi-conducteurs pixélisés présentent une excellente résolution en énergie, inférieure à 10\%, mais une résolution spatiale intrinsèque limitée par une taille des pixels de l'ordre de $2 \mathrm{~mm}$. À l'inverse, les dispositifs à scintillation offrent une bonne résolution spatiale intrinsèque, associée à une résolution énergétique dégradée supérieure à 15\%.

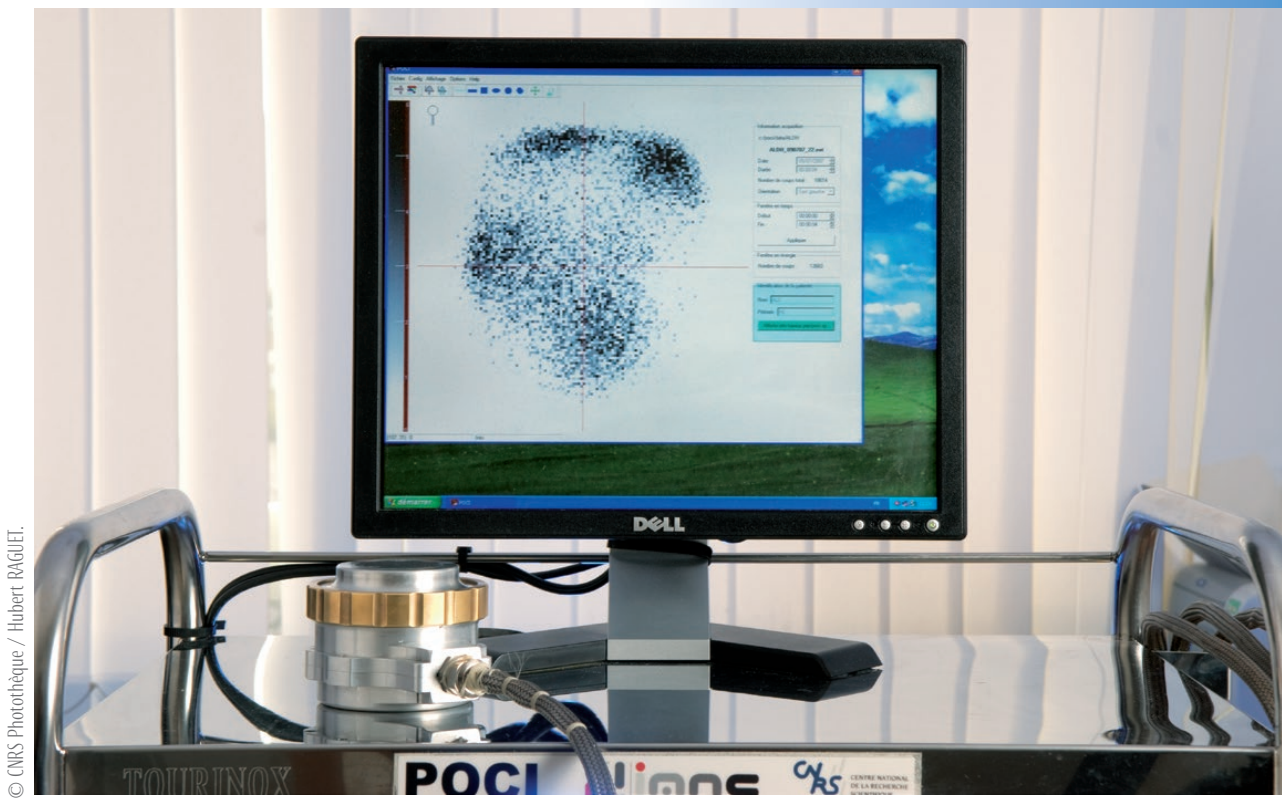

Si toutes les gamma-caméras miniaturisées présentent aujourd'hui des performances de détection proches, leurs géométries en revanche different. Ainsi, on distingue deux approches : les dispositifs avec des petits champs de vue inférieurs ou égaux à $5 \mathrm{~cm}$ de côté et ceux intermédiaires pour lesquels ce champ a une valeur de 10 à $20 \mathrm{~cm}$ de côté, à comparer aux champs de vue des gamma-caméras traditionnelles de $40 \times 50 \mathrm{~cm}^{2}$. La première approche offre l'avantage d'accéder à une grande compacité de la caméra, la rendant ainsi portable et donc facilement utilisable dans différents lieux (services de médecine nucléaire, blocs opératoires, mais également dans les chambres des patients); la petite surface d'analyse implique en contrepartie de réaliser un balayage rigoureux de la zone d'intérêt pour détecter la totalité des lésions. À l'inverse, la seconde approche instrumentale permet d'obtenir l'image d'une plus large région en un seul cliché ; néanmoins, de grandes dimensions empêchent de plaquer la tête de détection au contact de la peau du patient et compliquent le maniement de l'appareil, avec la présence indispensable d'un bras mécanique articulé.

Parmi les dix-neuf caméras existantes, seules onze ont été introduites en bloc opératoire pour réaliser des évaluations cliniques de la procédure de biopsie du ganglion sentinelle du cancer du sein, l'imagerie des adénomes parathyroïdiens, des cancers des os, des tumeurs cérébrales et enfin plus récemment des cancers colorectaux [1]. Les études cliniques rapportées aujourd'hui dans la littérature sur les gamma-caméras miniaturisées restent encore peu nombreuses et ont impliqué un nombre réduit de patients, inférieur à 60 . Dans ce contexte, l'étude menée avec la caméra POCI a été l'une des premières à avoir été initiée dès 2006 et reste à ce jour la plus importante à l'échelle internationale, avec une inclusion de 162 patientes [2].

\section{L'imageur per-opératoire POCI (Per-Operative Compact Imager)}

Le programme POCI a démarré en 1996 au sein du groupe Interfaces Physique Biologie de l'Institut de Physique Nucléaire d'Orsay (devenu depuis le laboratoire d'Imagerie et Modélisation en Neurologie et Cancérologie, UMR8165, CNRS Université Paris Sud - Université Paris Diderot), et visait à réaliser une gammacaméra miniaturisée pour l'assistance au traitement chirurgical du cancer.

Après le développement de plusieurs prototypes [3, 4], un nouveau prototype de la caméra POCI a été mis au point en 2005 pour être spécifiquement dédié aux essais cliniques sur l'homme, avec une certification de mise aux normes électromédicales. L'imageur POCI a été élaboré autour d'une tête de détection gamma de $4 \mathrm{~cm}$ de diamètre, constituée d'un collimateur multicanaux en plomb, d'un scintillateur CsI(Tl) et d'un système photosensible original associant un tube intensificateur d'image et une photodiode silicium à localisation bidimensionnelle ayant une grande surface sensible de 4,2 ×4,2 $\mathrm{cm}^{2}$ (voir l'encadré et la figure E1). La mise en œuvre d'une photodiode silicium à jonction PN garantit à la fois une simplicité de lecture avec seulement quatre voies électroniques indépendantes et un encombrement réduit, tout en offrant une grande surface d'analyse. 
$>>$

Par ailleurs, la photodiode silicium présente de très bonnes performances spatiales, grâce à son couplage au tube intensificateur d'image qui amplifie plus de 500 fois le nombre de photons issus du scintillateur. Cette solution instrumentale représentait en 2005 le meilleur compromis entre performances de détection, faibles dimensions externes et facilité de la mise en œuvre de l'électronique d'acquisition. offre un champ de vue de $4 \mathrm{~cm}$ de diamètre, pour des dimensions externes réduites $\left(9,5 \times 9,5 \times 9,0 \mathrm{~cm}^{3}\right)$ et une masse de $1,2 \mathrm{~kg}$ (fig. 1). Les performances de la caméra POCI ont été évaluées à partir d'une solution de nanocolloïdes de sulfure de rhénium marqués au ${ }^{99} \mathrm{~m}$ Tc (radio-isotope émetteur gamma à $140 \mathrm{keV}$, principalement utilisé en médecine nucléaire pour l'imagerie gamma). La résolution spatiale au contact du collimateur est de $2,2 \mathrm{~mm}$, ce qui est très inférieur à celle des gamma-caméras
Le prototype actuel de la caméra POCI

utilisées dans les services de médecine nucléaire (environ $6 \mathrm{~mm}$ ). L'efficacité de détection correspondante est de 0,028\%, ce qui permet une acquisition d'image en quelques secondes pour les applications cliniques courantes, avec des injections d'environ $50 \mathrm{MBq}$. Une telle sensibilité, associée à une surface d'analyse de $13 \mathrm{~cm}^{2}$, permet d'explorer une plaie opératoire dans un temps compatible avec la durée d'une intervention chirurgicale d'ablation tumorale.

C'est à partir de ce nouveau dispositif que l'intérêt des systèmes d'imagerie gamma per-opératoire dédiés au traitement chirurgical du cancer a été évalué. Pour cela, un Programme Hospitalier pour la Recherche Clinique (PHRC) national a été initié, en collaboration avec le service de Gynécologie Obstétrique de l'Hôpital Tenon (Paris), pour évaluer la caméra POCI. La procédure de radioguidage

\section{Principe de fonctionnement de la gamma-caméra POCI}

Parmi les photons gamma émis par la lésion tumorale radiomarquée, certains arrivent sur le collimateur avec une incidence et une position telles qu'ils vont le traverser, suivant une direction qui permet d'estimer la ligne sur laquelle ils ont été émis. Le collimateur est en plomb et est constitué d'un réseau hexagonal de canaux (fig. E1) de 1,4 mm de diamètre et de $15 \mathrm{~mm}$ de longueur, ce qui confère à la caméra $\mathrm{POCl}$ une résolution spatiale de $2,2 \mathrm{~mm}$ et une efficacité de détection de $0,028 \%$. Le dépôt d'énergie de la particule gamma dans le scintillateur crée un spot lumineux local, qui est collecté par proximité sur la fenêtre d'entrée du tube intensificateur d'image. Celui-ci amplifie la brillance du spot, tout en préservant l'information spatiale et temporelle, et l'achemine jusqu'à la photodiode à localisation bidimensionnelle. Cette dernière fournit simultanément la position du barycentre et l'énergie totale du paquet de photons ayant interagi dans la zone de déplétion par division de charge. Ainsi, chaque événement gamma est caractérisé à la fois par sa position dans un plan et par son énergie cédée dans le scintillateur. Les signaux détectés sont traités puis amplifiés par un système de lecture, pour être finalement transférés vers un ordinateur afin d'être numérisés et visualisés en temps réel sur l'écran.

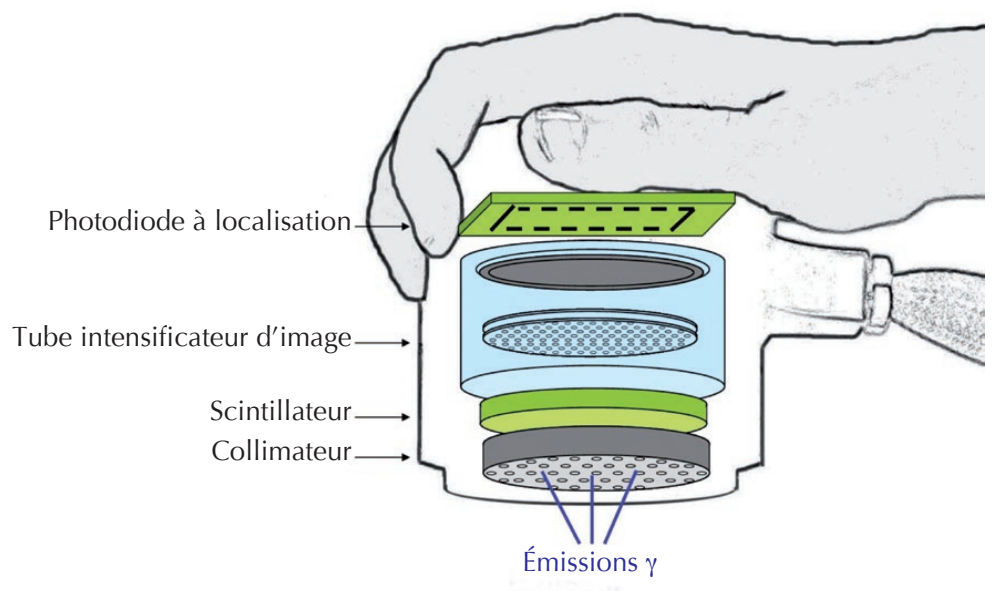

E1. Schéma de principe de la caméra P0Cl. retenue pour cette étude clinique était la biopsie du ganglion sentinelle dans le cadre des cancers du sein, qui constitue actuellement la procédure la plus largement décrite dans la littérature. Ce protocole clinique consiste à pratiquer un curage ganglionnaire uniquement en cas d'envahissement métastatique du premier ganglion, appelé " ganglion sentinelle ", chez des patientes atteintes d'une tumeur solide. Comme la localisation du ganglion sentinelle est imprévisible, un protocole de radiodétection a été établi selon deux étapes successives : i) l'identification et la localisation transcutanée du ganglion radiomarqué par scintigraphie ${ }^{\star}$, puis ii) son repérage et son prélèvement chirurgical assisté par radioguidage. Dans la pratique, après l'injection d'une solution radioactive de nanocolloïdes de sulfure de rhénium marqués au ${ }^{99 \mathrm{~m}} \mathrm{Tc}$ autour de la tumeur, une scintigraphie des aires ganglionnaires $\star$ est réalisée dans un service de médecine nucléaire à l'aide d'une gamma-caméra standard. Cette première étape permet de dénombrer les ganglions qui fixent la radioactivité (en moyenne deux par patiente) et de les localiser précisément. Ils sont ensuite biopsés en bloc opératoire avec l'aide de la sonde de comptage. La seconde étape de détection per-opératoire permet au chirurgien de vérifier la position du ganglion sentinelle avant l'incision, puis de l'identifier dans la plaie opératoire et enfin, après excision, de confirmer l'absence de toute radioactivité résiduelle.

Dans ce contexte, le protocole d'étude de la caméra POCI visait à évaluer la capacité de l'appareil lors des deux étapes de la radiodétection : i) pour réaliser une scintigraphie des aires ganglionnaires et ii) pour guider le geste chirurgical en complément des sondes de comptage. Pour cela, une double étude clinique prospective a été menée à l'aveugle, en intégrant l'imageur POCI au protocole standard de la détection $\mathrm{du}$ ganglion sentinelle : POCI versus la gamma-caméra standard et POCI versus la sonde de comptage. Dans le premier cas, il s'agissait d'évaluer les performances de l'imageur POCI pour établir une cartographie précise des aires de drainage ganglionnaire et de comparer le nombre et la localisation des ganglions sentinelles (fig. 2B) ainsi détectés à ceux obtenus avec la gammacaméra clinique standard (fig. 2A). La seconde partie de l'étude visait à déterminer l'apport de l'imageur POCI en complément de la sonde de comptage. Le chirurgien examinait 

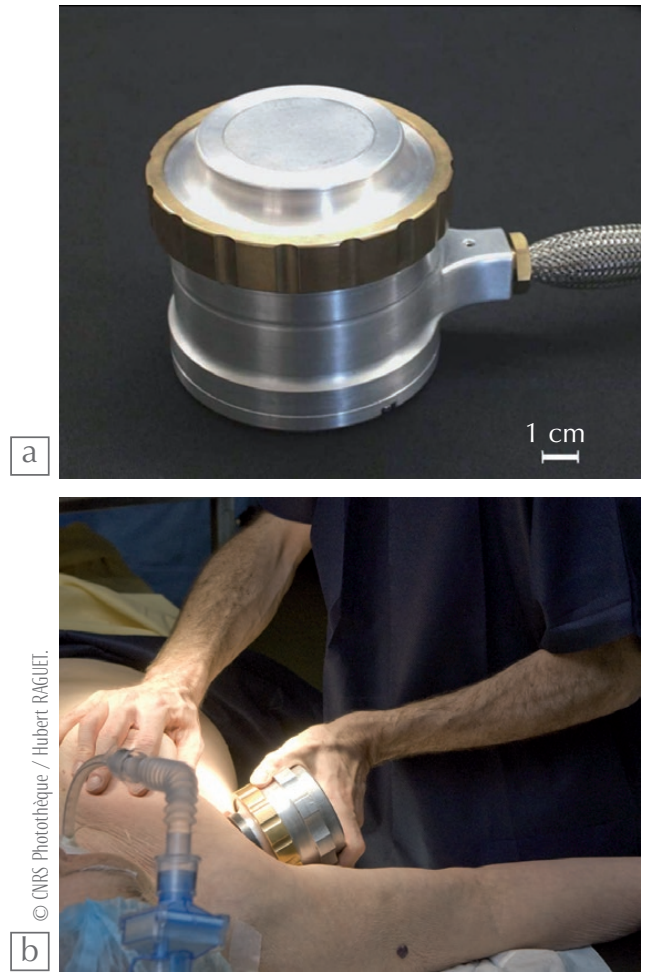

1. La caméra POCI (a) et son utilisation en bloc opératoire (b) lors de la localisation transcutanée des ganglions sentinelles.

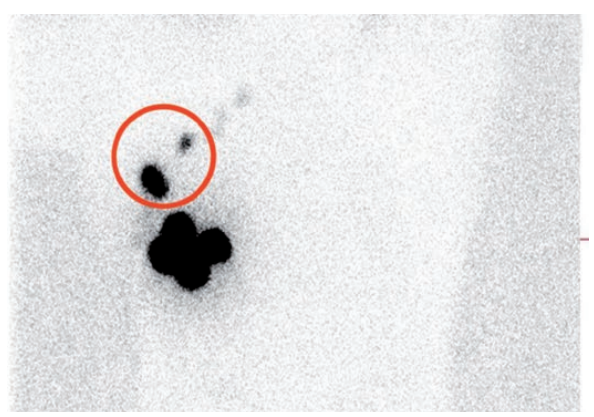

b

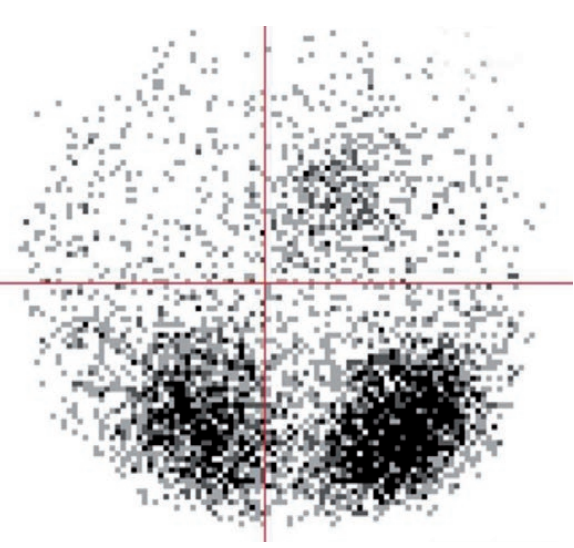

2. Scintigraphies comparées. (a) Cliché scintigraphique obtenu en 5 minutes avec une gamma-caméra standard, et révélant à l'intérieur du cercle rouge la présence de deux ganglions fixant la radioactivité. (b) Image de la zone correspondante, obtenue en 22 secondes avec la caméra POCl et permettant d'identifier la présence de trois ganglions. alors la capacité des deux détecteurs à fournir une localisation et un dénombrement exact des ganglions dans la plaie opératoire en l'absence de scintigraphie préliminaire.

Avec 162 patientes incluses, cette étude clinique est aujourd'hui la plus significative statistiquement sur le plan international. Ses résultats ont montré pour la première fois, qu'un dispositif d'imagerie gamma miniaturisé était "non inférieur " à une gamma caméra standard pour sa capacité à dénombrer les ganglions sentinelles et ce, avec des temps d'acquisition particulièrement courts : seulement huit minutes avec l'imageur POCI pour parcourir manuellement les aires ganglionnaires, contre quinze minutes avec la gamma-caméra standard pour réaliser en routine trois clichés d'incidences différentes (cinq minutes d'acquisition par incidence). En bloc opératoire, seule la région axillaire (au niveau de l'aisselle) était à explorer. Ainsi, le temps d'examen de la zone chirurgicale avec la caméra POCI était de trois minutes en moyenne, durée compatible avec une utilisation per-opératoire de l'appareil. Au cours de l'intervention chirurgicale, l'imageur POCI a permis la détection de 21 ganglions radiomarqués supplémentaires non repérés par la sonde de comptage chez 20 patientes, soit dans $15 \%$ des cas. Parmi ces 21 ganglions, cinq se sont révélés métastatiques et ont conduit à une modification de la stratégie thérapeutique envisagée pour les patientes.

\section{Glossaire}

Détecteur per-opératoire : détecteur dédié à une utilisation au cours de l'intervention chirurgicale.

Collimateur sténopé ou "pinhole" : ce collimateur présente un seul trou de faible diamètre, permettant d'obtenir l'image de la scène radioactive avec un facteur de grandissement.

Aires ganglionnaires : dans le cas du cancer du sein, les aires ganglionnaires concernées regroupent trois chaînes ganglionnaires axillaire au niveau de l'aisselle, sus-claviculaire, et enfin mammaire interne, dont les ganglions se situent de 2 à $4 \mathrm{~cm}$ de l'axe médian du sternum.

Scintigraphie : examen réalisé à l'aide d'une gamma-caméra.

\section{Conclusion}

L'imageur POCI a permis de valider l'intérêt des gamma-caméras miniaturisées, tant pour remplacer les caméras conventionnelles pour l'imagerie de régions anatomiques réduites que pour compléter les sondes de comptage lors du traitement chirurgical du cancer. Ce protocole suscite un grand intérêt de la part de la communauté médicale, puisqu'il concerne la détection des lésions inférieures à $1 \mathrm{~cm}^{3}$, de plus en plus fréquente en raison du dépistage systématique et précoce du cancer du sein.

Au-delà du ganglion sentinelle du cancer du sein, d'autres protocoles peuvent également tirer profit d'un système d'imagerie peropératoire, comme l'assistance au traitement chirurgical des tumeurs mammaires non palpables. En plus de la détection transcutanée du ganglion, l'imageur pourrait intervenir au cours de ce protocole chirurgical pour guider le geste d'ablation en temps réel de la tumeur préalablement radiomarquée et vérifier la qualité du geste opératoire par une simple image scintigraphique.

En termes d'évolution instrumentale, le développement récent de Si-PM (Silicium PhotoMultiplier) comme photodétecteur peu encombrant, ouvre la voie à une nouvelle génération de gamma-caméras ultra-compactes [5].

\section{Références}

1• M. Tsuchimochi et K. Hayama, "Intraoperative Gamma Cameras for Radioguided Surgery: Technical Characteristics, Performance Parameters, and Clinical Applications", Phys. Med., 29 (2013) 126-138.

2-K. Kerrou et al., "The Usefulness of a Preoperative Compact Imager, a Hand-Held Gamma-Camera for Breast Cancer Sentinel Node Biopsy: Final Results of a Prospective Double-Blind, Clinical Study", J. Nucl. Med., 52 (2011) 1346-1353.

3• L. Ménard et al., "Performance Characterisation and First Clinical Evaluation of an Intra-0perative Compact Imager", IEEE Trans. Nucl. Sci., 46 (1999) 2068-2074

4- S. Pitre et al., "A High Resolution Hand-Held Gamma Camera for Cancer Surgery”, Journal of Nuclear Medicine, 42 (suppl.) (2001) 202P.

5• S. Yamamoto et al., "Development of a high-resolution Si-PM-based gamma camera system", Phys. Med. Biol., 56 (2011) 7555-7567. 